\title{
曷Digital Publisher
}

ISSN 2588-0705

Modelos administrativos y organización de MIPYMES de Machala como estrategia de crecimiento al mediano plazo

Administrative models and organization of MIPYMES in Machala as a strategy for mediumterm growth

Ayrton David Ortega-Reyes Universidad Técnica de Machala - Ecuador aortega2@utmachala.edu.ec

Ruth Esther Samaniego-Serrano Universidad Técnica de Machala - Ecuador rsamanieg2@utmachala.edu.ec

doi.org/10.33386/593dp.2021.6.722

V6-N6 (nov-dec) 2021, pp. 36-48 | Recibido: 13 de agosto de 2021 - Aceptado: 01 de septiembre de 2021 (2 ronda rev.) 
Cómo citar este artículo:

APA:

Ortega-Reyes, A., \& SamaniegoSerrano, R., (2021). Modelos administrativos y organización de MIPYMES de Machala como estrategia de crecimiento al mediano plazo. 593 Digital Publisher CEIT, 6(6), 36-48. https://doi. org/10.33386/593dp.2021.6.722

Descargar para Mendeley y Zotero

\section{RESUMEN}

Muchas empresas que buscan el crecimiento tratan de tomar como ejemplo modelos administrativos que se ajusten a su realidad para poder dirigir de forma eficiente sus esfuerzos y de esta forma obtener mejores resultados al mediano y largo plazo. El presente estudio determina si los modelos administrativos y la organización empresarial influyen en el crecimiento al mediano plazo en las MIPYMES de la ciudad de Machala. La presente investigación es de carácter cualitativo de tipo descriptiva-transversal, para su desarrollo se aplicó una encuesta en donde se consideró un total de 375 Micro, pequeñas y medianas empresas que desempeñan actividades comerciales y de servicios con la finalidad de analizar su forma de administrar. Los resultados determinaron que la mayoría de las Mipymes de la ciudad de Machala presentan inconvenientes al momento de organizarse y desconocen de los diferentes modelos administrativos que se pueden aplicar según su necesidad, esto impide el crecimiento y cumplimiento de los objetivos organizacionales.

Palabras clave: administración; crecimiento; MIPYMES; organización

\section{ABSTRACT}

Many companies that seek growth try to take as an example, administrative models that fit their reality to be able to efficiently direct their efforts and thus obtain better results in the medium and long term. This study determines whether administrative models and business organization influence medium-term growth in MSMEs in the city of Machala. The present research is of a qualitative nature of descriptive-transversal type, for its development a survey was applied where a total of 375 Micro, small and medium enterprises that carry out commercial and service activities were considered to analyze their way of administering. The results determined that most of the MSMEs in the city of Machala have inconveniences when organizing and are unaware of the different administrative models that can be applied according to their need, this prevents the growth and fulfillment of organizational objectives.

Key words: administration; growth; MSMEs; organization 


\section{Introducción}

Las empresas a nivel global constituyen el mayor porcentaje de actividad económica, siendo estas las que aportan a las economías de los países convirtiéndolos en potencias mundiales, es importante destacar que las empresas requieren de la capacidad humana para llevar a cabo las diferentes actividades, que de la mano con la tecnología van creando así plazas de trabajo y aportan a la mejora de la economía. En Latinoamérica las MIPYMES sobresalen en especial por su gran importancia en el sector económico y generación de empleo, aunque generalmente manejan un sistema informal buscan nuevas oportunidades en el mercado (CEPAL, 2018).

El Ecuador se encuentra representado en un $75,00 \%$ por empresas pequeñas y medianas que a su vez aportan a la economía generando más del 70,00\% de plazas de trabajo (Cervantes \& Oviedo, 2018). Las mipymes enfrentan constantes desafíos que pueden llegar a constituir fuertes amenazas a su sobrevivencia entre ellos se encuentra el aprendizaje y la práctica de nuevas formas de administración, así como la reinvención para transformase de una empresa doméstica, a una gran empresa para lo cual es necesario realizar cambios fundamentales en su forma de administrar entrando en un proceso de integración y automatización de la información y de procesos para volverse eficientes (Cervantes \& Oviedo, 2018).

En El Oro, las mipymes son representativas en el sector productivo y generador de empleo, donde el 99,7 están distribuidas en micro el $89,5 \%$, pequeñas $(8,4)$ y medianas $(1,8)$ empresas. (INEC, 2015). Para mejorar la competitividad es indispensable que las empresas desarrollen ventajas competitivas que generen beneficios sobre las mismas, esto abarca conocer el mercado en el que se desarrolla, los clientes a los que dirige su producto y toda la información válida y relevante sobre rentabilidad que llevarán al correcto funcionamiento de la empresa. (Luciani, Zambrano, \& Gonzalez, 2019).
La presente investigación es de carácter cuantitativo de tipo descriptiva-transversal, esto quiere decir que se procederá a describir las características administrativas de cada tipo de empresa y mediante la aplicación de un cuestionario se hará un análisis comparativo entre las formas de administrar y de esta forma determinar si la estructuración empresarial resulta clave para el crecimiento al mediano plazo.

El objetivo del presente trabajo de investigación es determinar si los modelos administrativos y la organización influyen en el crecimiento al mediano plazo de las micro y medianas empresas, mediante el análisis del comportamiento de diferentes Mipymes de la ciudad de Machala con el fin de identificar si dichas características corresponden a un factor clave para el crecimiento al mediano plazo. Así mismo para su cumplimiento se definirán los modelos administrativos $\mathrm{y}$ formas de organización que se puedan aplicarse por las microempresas, se analizará el comportamiento de las microempresas mediante la aplicación de una encuesta para identificar sus formas de trabajo vs sus logros obtenidos y se establecerá si es necesario implantar nuevos modelos de gestión y reorganización de las microempresas para alcanzar su crecimiento a mediano plazo.

\section{Desarrollo}

\section{Administración}

Administración empresarial es un proceso coordinado de un equipo de personas que tienen un objetivo en común, alcanzar los objetivos empresariales de la mano con las estrategias que aporten a su realización. De acuerdo a los procedimientos que se apliquen en las empresas, siempre relacionadas con la actividad comercial que realicen, se dará cumplimiento a los resultados que se desean alcanzar (Arteaga, Intriago, \& Mendoza, 2016). 


\section{Funciones de la administración}

El autor Veliz (2018) señala que, con la finalidad de apoyar a los líderes de organizaciones en sus actividades, se han creado funciones principales que cumple la administración de empresas, entre las cuales sobresalen la planificación, la cual se conceptualiza como la fase principal que el administrador toma en consideración para aplicar en sus actividades dentro de la empresa; La organización, hace referencia al diseño de las formas en las que se da cumplimiento a los objetivos empresariales además de llevar un orden en toda la organización; La dirección, comprende los canales de comunicación, liderazgo y manejo de conflictos en la empresa, además de motivar al personal al cumplimiento de objetivos y por último el control que es el proceso de seguimiento y verificación del correcto cumplimiento de actividades en la organización.

\section{Diseño organizacional}

El diseño organizacional corresponde al conjunto integrado de la estructura de la organización, procesos, obligaciones y las interacciones que se suscitan dentro de la organización, en donde también se analizan los cambios que se dan en el exterior las cuales influyen dentro de la empresa, todo ello para lograr eficiencia y eficacia dentro de la empresa y a su vez cumplir con los objetivos que esta halla planificado, sin dejar a un lado el control sobre dichas actividades (Navarro, Cota , \& González, 2018). Es por ello que el diseño organizacional resulta muy importante dentro de las empresas cuando se trata de fortalecer la calidad dentro de los procesos y la optimización tanto de los recursos como también de talento humano, por ello el buen diseño organizacional resultara estratégico dentro del desempeño de cada puesto laboral, esto debido a que proporciona mayor visión, y rutas para la toma de decisiones que puedan conducir a un beneficio (Hernández , Martinez, \& Barrios, 2018).
El diseño organizacional dentro de las empresas permite tener una mayor visión de la forma en la que opera la empresa, saber qué cargo ocupa cada persona, como trabaja, que acciones realiza, como se comunica y como participa, ayuda a mejorar la calidad del trabajo y permite la sinergia entre colaboradores, por ello el diseño organización resulta clave para el éxito, aunque no es muy conocido por muchas empresas. Para poder obtener un diseño organizacional que opere de forma óptima. Se debe contar con una buena estructura organizacional y a su vez cargos laborales que cumplan con funciones importantes y no redundantes, debe ser flexible y mantenerse alineados a los objetivos de la empresa, es decir, a sus estrategias, procesos y cultura con la esperanza de poder alcanzar mayor productividad e impacto dentro de la organización que se traducen en el alcance de los objetivos (Flores, 2017).

Se puede comprender entonces que gran parte del éxito se centra en saber realizar un buen diseño organización, que opere de forma eficiente, con una buena distribución de tareas y responsabilidades que permitan reducir la carga laboral, de esta forma optimizar recursos y alinear los objetivos a las necesidades de la empresa.

\section{Estructura organizacional}

La estructuración organizacional puede ser comprendida como la forma en la que se distribuye cada unidad dentro de una institución, basándose en la meta que busca conseguir la empresa con el fin de poder cumplirlos (Bernal, Erazo , \& Narváez, 2019). Desde otro punto de vista se puede comprender la estructuración como la forma de gestionar y coordinar cada una de las actividades de un puesto o lugar de trabajo, estas se pueden evidenciar en la departamentalización, la especialización dentro de cada puesto de trabajo o las líneas jerárquicas existentes dentro de una empresa, entre otras (Ocampo, Huilcapi, \& Cifuentes, 2019). 
En base a lo expresado con anterioridad se puede comprender que las empresas se organizan de forma diferente y esto se debe a que cada una busca el crecimiento de una forma diferente, cada una deberá dividir y organizar el talento humano con la intención de lograr la obtención de mayores recursos económicos, es por eso que existen diferentes formas de estructurar una empresa, por un lado existe aquella que se conoce como estructura vertical en la cual se establecen niveles jerárquicos en donde cada puesto busca cumplir cada una de las funciones requeridas del puesto y que son supervisadas y controladas por una persona de un nivel superior, por otro lado podemos ver la estructuración horizontal que por el contrario busca generar mayor compromiso por parte de los empleados en donde puedan participar con ideas y tomar decisiones en base a sus conocimientos (Ocampo, Huilcapi, \& Cifuentes, 2019).

\section{Modelos administrativos}

\section{Tradicional}

Este tipo de administración se caracteriza por la forma de manejar los procesos $\mathrm{y}$ actividades realizadas en la empresa, las mimas que son estricta y únicamente reguladas por el administrador. Al no manejar una administración flexible se vuelve casi imposible la adaptación del personal al entorno socioeconómico de la empresa ya que este no tiene mayor participación. La toma de decisiones en este tipo de administración también se ve afectado ya que se complica el tener una información precisa en la que basarse para evitar fallos en el proceso (Valarezo \& Coronel, 2018).

Por otra parte, Viloria y Luciani (2015) , señala que la administración tradicional se fundamenta en estudiaos realizados por los teóricos de la administración, los mismos que determinan que este tipo de administración se lleva a cabo basándose en determinismo. La administración tradicional lleva al cumplimiento de los objetivos con carencia de conocimiento además de llevar un entorno alejado a la realidad que enfrenta la empresa y sus actores principales, el talento humano.

\section{Estructurado}

La administración es también darle consistencia a la empresa, mediante el cumplimento de los objetivos y metas, los mismos que deben basarse en planes y estrategias que lleven a cabo su realización influyendo además a los trabajadores haciendo de la administración un proceso flexible y otorgador de resultados positivos. Las nuevas tendencias en la administración hacen que los procesos sean más eficaces y optimicen los recursos dando la posibilidad que la empresa sin importar su tamaño genere valor agregado a su bien o servicio brindado (Buñay, Montalvan, \& Vizueta Sonia, 2018).

La organización es parte de un entorno, donde participan recursos financieros, humanos y materiales, los mismos que deben estar conectados de manera que sus procesos sean eficientes y conseguir el rendimiento óptimo. La interrelación facilita la identificación de problemas y así mismo permite la rápida respuesta a los mismos, además de permitir a la empresa adaptarse al entorno en el que se desarrolla. La eficiencia y eficacia de la organización se mide en el nivel de satisfacción del consumidor cumpliendo así con las expectativas de los clientes internos y externos (Saltos, Muñoz, \& Rodriguez, 2016).

\section{Nuevas formas de organización Empresarial}

Con el contante crecimiento de la globalización y las nuevas formas de crear y diseñar las empresas, las lianas directivas deben comprender que las empresas deben volverse cada vez más competitivas, innovando, creando nuevos productos y así mismo mejorando la forma de trabajar, es por ello que a lo largo del tiempo surgen nuevas formas de organizar una empresa y nuevas estrategias para alcanzar resultados, Es por ello que se debe debatir sobre que formas de trabajo deben mejorarse y mantenerse, así mismo las organizaciones tradicionales están buscando en convertirse organizaciones más flexibles, a través de la promoción del trabajo en equipo, incorporación de nuevas ideas, además de promover la responsabilidad y la unión, de 
esta forma ir hacia un mismo fin ( Vega, 2016).

Actualmente han surgido muchas formas nuevas de administrar, formas que han analizado la administración tradicional y que han incursionado a la innovación tanto técnica como de gestión, logrando de esta forma tener un mayor desempeño en la empresa y por ende mejores resultados (Vega \& Salaiza, 2016).

Dentro de los principales aportes dentro de las alternativas para la eficiente administración se encuentran la evasión de las jerarquías a través de una estructura organizacional horizontal, la descentralización de la empresa y la capacidad de tomar decisiones de forma más autónoma, estas nuevas formas de administrar buscan manejar los retos que día a día presentan las empresas y de esta forma adquirir no solo una ventaja competitiva sino también dominar la incertidumbre dentro la empresa, aumentar la capacidad de producir ideas, reducir de forma gradual los costos, aliarse con las tecnologías de la información y poder diseñar un ambiente que sea más ágil con capacidad de respuesta inmediata (Vega \& Salaiza, 2016).

\section{Tipos de empresas}

\section{Microempresas}

Sus ventas anuales son menores o iguala a $\$ 100.000$ y sus trabajadores son de 1 hasta 9 . A nivel mundial, especialmente en Latinoamérica esta categoría de empresas son bastante numerosas y según estudios realizados son negocios o emprendimientos que son impulsados por factores como la necesidad y desempleo, generalmente el dueño del emprendimiento constituye la motivación más importante, sus colaboradores no son numerosos y cumplen funciones con poco valor agregado (Texis, Ramirez, \& Aguilar, 2016).

\section{Pequeñas empresas}

Estas empresas tienen sus ventas anuales desde $\$ 1000.000$ y las fuentes de empleos van desde 10 a 49 (INEC, 2019). Estas empresas son numerosas en el mercado, aunque sus inversiones en innovación son mínimas además que en algunos casos esta inversión no tiene un previo estudio o planificación. Para su innovación estas empresas requieren capacidades humanas y tecnológicas, lo que se ha convertido en un reto para esta clasificación de empresas (Sanchez, Cervantes, \& Peralta, 2016).

Las MIPYMES en América Latina son fundamentales para el desarrollo económico, ya que además de aportar a la economía del así son fuente generadora de trabajo ara los ciudadanos, en algunos países las actividades de las mipymes en el mercado representan gran porcentaje del producto interno bruto. Las mipymes se caracterizan por la especialización en bienes o servicios con un bajo valor agregado, además de su participación reducida en mercados internacionales (Larios, 2016). Mipymes, en Ecuador aproximadamente 99 de cada 100 empresas son mipymes (microempresas, pequeñas y medianas empresas), esto significa que esta categoría abarca el mayor sistema productivo en el país (Quispe, Padilla, Telot, \& Nogueira, 2017)

\section{Grandes empresas}

El tamaño de la empresa se define según el nivel de ventas anuales que tenga, además del número de trabajadores o plazas de empleo que genere, dentro de la categoría grande empresa se la denomina así ya que sus ventas son de $\$ 5000001$ o más y el número de empleados van desde 200 en adelante. Las grandes empresas son menos en número que las otras pero su aporte económico al país es alto en comparación los otros tipos de empresas (INEC, 2019).

\section{Factores que inciden en el bajo rendimiento de las empresas}

La falta de crecimiento dentro de las MIPYMES se considera como un problema grave que surge como consecuencia de no llevar una buena administración, parte importante de este bajo crecimiento es la falta de eficiencia en las empresas, el desconocimiento de su mercado meta o público objetivo, la falta de capacidad para liderar, un marco legal que no se encuentre actualizado y la poca información 
acerca del entorno sobre la cual se desarrolla (Armijos, 2019). Desde otro punto de vista Las causas de dicha falta de crecimiento se deben a factores como falta de recursos económicos, falta de controles sobre las empresas, problemas vinculados con la gestión como precios excesivamente bajos, alta rotación de puestos de trabajo y un tamaño de empresa que no se apega a los ingresos de esta (Baque, Chele, Cedeño, \& Gaona, 2020).

Así mismo podemos Agregar otros factores que hacen referencia al empresario tales como el exceso de carga laboral, la mala delegación de actividades o funciones y la experiencia profesional (Baque, Chele, Cedeño, \& Gaona, 2020). El crecimiento de una empresa puede ser muy deseado, pero se necesita de visión para poder lograr que una empresa pueda crecer y sobretodo mantenerse durante el tiempo, es por ello que diariamente muchos empresarios y emprendedores buscan formas y herramientas que les permitan sobrevivir y poder mirar hacia el largo plazo con la intención de poder genera mayores ingresos y alcanzar una cuota de mercado más amplia.

\section{Modelos de gestión administrativa}

\section{Modelo de Malcom Baldrige}

La correcta administración depende en gran parte de la colaboración de los empleados en el mejoramiento de la empresa, esto se lleva a cabo a través de la formación y capacitación del personal para que se involucren dentro de la misma. Dentro del modelo en mención existen siete criterios, los cuales son el liderazgo, planteamiento estratégico, dirección hacia el consumidor y el mercado, la gestión del conocimiento, enfoque al personal, gestión de los procesos y la presentación de resultados.

En conclusión, este modelo se orienta en dos puntos, la dirección de la empresa y la satisfacción de los clientes mejorando las relaciones internas de la empresa generando mayor productividad y valor para todas las partes involucradas generando nuevas capacidades en los empleados (Silva, Feijó, \& Feijoo, 2020).

\section{Modelo Deming}

Según Recalde et al. (2017) este modelo fue adaptado por primera vez en Japón recogiendo conceptos de diferentes teorías para lograr el mejor control de calidad empresarial, tiene como objetivo que la empresa trabaje en conjunto, orientan a la satisfacción de necesidades de los clientes actuales y potenciales y así lograr los objetivos y metas deseados.

Los elementos que intervienen en este modelo son la planificación, ejecución, evaluación y actuación, dentro de dichos elementos se establecen las metas, se pone en práctica aquello que se propone, se miden los resultados obtenidos mediante la implantación y desarrollo de cada objetivo, por último, se realiza una retroalimentación considerando las fallas y puntos a favor dentro del modelo para poder establecer una mejora continua.

\section{Modelo Servqual}

Para Pincay y Parra (2020) el modelo Servqual permite realizar una medición con respecto a la calidad del servicio al cliente, dicho modelo busca valorar las diferencias existentes entre las expectativas de los productos o servicios y la percepción de los clientes al momento de adquirir dichos productos. Así mismo, el modelo propone diferentes dimensiones que permiten la evaluación del servicio recibido las cuales son la confianza entre la empresa y el cliente, la imagen corporativa y la seguridad, la capacidad de respuesta frente a las diferentes necesidades y por último los aspectos tangibles.

El modelo en mención resulta muy útil a la hora de conocer de qué forma los clientes perciben la calidad del servicio de atención al cliente. Muchas empresas en la actualidad realizan un estudio sobre la calidad de su servicio con la esperanza de cumplir con las expectativas de sus clientes logrando la fidelización de estos asegurando compras futuras y con ello la permanencia de la empresa. 


\section{Método}

El enfoque que posee la investigación en mención es cualitativo de tipo descriptivatransversal, mediante el desarrollo de misma se busca caracterizar y determinar cuáles son los modelos de administración que aplican los diferentes tipos de Mipymes en la ciudad de Machala, de esta forma analizar si el crecimiento en las empresas se ve afectado por su forma de administrar (Hernández , Fernández, \& Baptista, 2014). Para ello se consideró a la ciudad de Machala como población de estudio por ser la ciudad con mayor desarrollo de la provincia de El Oro y mayor concentración de empresas.

Según (INEC, 2015), el número de establecimientos económicos que hay en la ciudad de Machala es de 11.215, las cuales se encuentran distribuidas principalmente en actividades productivas, económicas y de servicio. Para Taillacq et al. (2016) la fórmula para el cálculo de la muestra de población finita es:

$$
n=\frac{N * Z^{2} * p * q}{e^{2} *(N-1)+Z^{2} * p * q}
$$

En donde:

$\mathrm{n}=$ tamaño de la muestra.

$\mathrm{N}=$ tamaño de la población.

$Z$ = parámetro estadístico que dependerá del nivel de confianza $(95 \%=1.96)$.

$\underline{\underline{\mathrm{e}}}=$ error máximo aceptado.

$\mathrm{p}=$ probabilidad de tener éxito.

$q=(1-p)$ probabilidad de que no tener éxito.

Para la realización del cálculo de la presente investigación se ha considerado un nivel de confianza del 95\% y los datos que se muestran a continuación:

\begin{tabular}{|c|c|}
$\mathbf{N}$ & 11.215 \\
\hline $\mathbf{Z}$ & 1,96 \\
\hline $\mathbf{p}$ & 0,50 \\
\hline $\mathbf{q}$ & 0,50 \\
\hline $\mathbf{e}$ & 0,05 \\
\hline
\end{tabular}

$$
\begin{aligned}
& n \\
& =\frac{11.215 * 1.96^{2} * 0.5 * 0.5}{0.05^{2} *(11.215-1)+1.96^{2} * 0.5 * 0.5} \\
& =\frac{10.770,89}{29,00}=372
\end{aligned}
$$

A través de la aplicación del cálculo de población finita, se determinó que la muestra será de 372 empresas, a las cuales se les aplicará una encuesta partiendo de preguntas generales las cuales son, sector económico al que pertenecen y la actividad que realiza sea esta comercial o de servicios, seguido de 22 preguntas divididas en componentes etapas, organización interna (planificación, supervisión y control de actividades, compromiso laboral, filosofía empresarial, procesos administrativos, objetivos a mediano plazo, estructura organizacional y toma de decisiones), estrategias empresariales (alcance de objetivos, oportunidad de financiamiento, diversificación de productos, mercado meta, seguimiento a clientes e inversión en marketing), evolución empresarial (talento humano, crecimiento empresarial, cartera de clientes), finalmente capacidad administrativa (modelos administrativos, asesoría administrativa, conocimientos administrativos, fuentes de financiamiento). Dichos componentes fueron seleccionados con la finalidad de analizar la forma en la que operan las empresas y su organización para determinar si estas influyen en el crecimiento a mediano plazo. 


\section{Análisis de resultados}

Los cuestionarios se realizaron a través de la plataforma google formularios y fueron enviados por correo electrónico a diferentes empresas de la ciudad de Machala utilizando datos que se obtuvieron a través del sistema de la Superintendencia de compañías, además se realizaron visitas a varias empresas para cumplir con la muestra requerida. Se logró obtener un total de 375 encuestas respondidas y se procedió a realizar el respectivo análisis, considerando aquellas preguntas que presentan mayor relevancia para el cumplimiento de los objetivos del estudio.

\section{Tabla 1}

Ítems de control y gestión

\begin{tabular}{lrr}
\hline & Número & Porcentaje \\
Actividades & 73 & $19,40 \%$ \\
Recursos & 88 & $23,30 \%$ \\
Inventario & 252 & $66,80 \%$ \\
Personal & 195 & $51,70 \%$ \\
Ningún control & 27 & $7,20 \%$ \\
\hline
\end{tabular}

Como se pudo apreciar en la tabla 1, de todos los ítems establecidos se aplica más aquellos destinados al control y gestión de inventarios con un $66.80 \%$ de aplicación y al personal con un $51.70 \%$ de aplicación, mientras que el control y gestión de actividades y recursos poseen un bajo porcentaje de aplicación.

\section{Tabla 2}

\section{Filosofía empresarial}

\begin{tabular}{lrr}
\hline & Número & Porcentaje \\
Misión & 46 & $12,20 \%$ \\
Visión & 39 & $10,3 \%$ \\
Estrategias & 87 & $23,1 \%$ \\
Políticas & 102 & $27,1 \%$ \\
Otras & 46 & $12,2 \%$ \\
Ninguna & 190 & $50,4 \%$ \\
\hline
\end{tabular}

Al analizar los resultados de la tabla 2 se puede identificar que las empresas encuestadas indican que el 12,20\% de ellas cuentan con misión empresarial, $10,3 \%$ cuentan con visión, $23,1 \%$ indico que contienen estrategias empresariales, $27,1 \%$ políticas, por otra parte, el $12,2 \%$ considera otros elementos y finalmente la mayoría con el 50,4\% indicó no cuenta con filosofía empresarial.

Tabla 3

Logro de objetivos

Número Porcentaje

Se han

logrado

cumplir sin

problemas

51

$13,5 \%$

Se han

presentado

problemas

en el

cumplimiento

224

$59,4 \%$

No se han

logrado

cumplir

$27,1 \%$

Total

$100 \%$

Dados los resultados de la tabla 3, se puede determinar que la mayoría de empresas con un $59,4 \%$ indica haber presentado problemas en el cumplimiento de objetivos, seguido del $27,1 \%$ que señala no haber cumplido sus objetivos.

Tabla 4

Modelos administrativos

\begin{tabular}{lrr}
\hline & Número & Porcentaje \\
Si conoce & 32 & $8,5 \%$ \\
Conoce poco & 127 & $33,7 \%$ \\
No conoce & 218 & $57,8 \%$ \\
Total & 377 & $100 \%$ \\
& &
\end{tabular}


Los resultados obtenidos en la tabla 4 dan a conocer que los encuestados con un $57,8 \%$ indican no tener conocimiento acerca de modelos administrativos, el $33,7 \%$ conoce poco del tema y el $8,5 \%$ señala si conocer de modelos administrativos.

\section{Tabla 5}

\section{Crecimiento empresarial}

\begin{tabular}{lrr}
\hline & Número & Porcentaje \\
Ha crecido & 57 & $15,1 \%$ \\
$\begin{array}{l}\text { Se ha mantenido } \\
\text { igual }\end{array}$ & 213 & $56,5 \%$ \\
$\begin{array}{l}\text { Tiene problemas } \\
\text { para mantenerse }\end{array}$ & & \\
$\begin{array}{l}\text { estable } \\
\text { Se encuentra en }\end{array}$ & 98 & $26 \%$ \\
declive & 9 & $2,4 \%$ \\
Total & 377 & $100 \%$
\end{tabular}

En cuanto a crecimiento empresarial las empresas encuestadas otorgaron resultados de la siguiente manera, el $15.1 \%$ de las empresas ha crecido empresarialmente, el $56,5 \%$ se ha mantenido igual, el $26 \%$ tienen problemas para mantenerse estable y finalmente el $2,4 \%$ de los encuestados manifiesta encontrarse en declive.

\section{Tabla 6}

\section{Cartera de clientes}

\begin{tabular}{lrr}
\hline & Número & Porcentaje \\
Ha crecido & 58 & $15,4 \%$ \\
Se ha & & \\
mantenido & & \\
igual & 250 & $66,3 \%$ \\
Ha disminuido & 69 & $18,3 \%$ \\
Total & 377 & $100 \%$
\end{tabular}

Según resultados de la tabla 6 , la cartera de clientes en las empresas encuestadas se desglosa de la siguiente forma, $15.4 \%$ de estas ha crecido, el $66,3 \%$ se ha mantenido igual, $y$ finalmente el $18,3 \%$ ha disminuido su cartera de clientes.

\section{Discusión}

En base a los resultados obtenidos mediante encuesta aplicada a la mipymes de la ciudad de Machala, se ha detectado que es significativo el número de empresas en su mayoría de actividad comercial, que carecen de estructuración, interviniendo varios factores importantes como la falta de una planificación diaria de sus actividades.

Así mismo otro factor es la falta de una filosofía empresarial clara en la mayoría de las empresas, existen limitaciones dentro del cumplimiento de objetivos, adicionalmente el desconocimiento de modelos de gestión empresarial implica que las mipymes no poseen lineamientos para organizarse debidamente y orientar su forma de trabajo a la consecución de sus objetivos.

Otro factor determinante es la evolución de la empresa en cuanto a rentabilidad, personal y cartera de clientes, los cuales en los tres indicadores se inclinan a que sus empresas se han mantenido igual en el mercado en torno a su giro comercial, así mismo se dio a conocer la dificultad de acceso a financiamientos, finalmente la falta búsqueda de nuevos mercados, y la inexistencia en muchos casos de un seguimiento postventa a sus clientes y la limitada inversión en marketing por parte de las organizaciones. todos corresponden a elementos importantes y determinantes del crecimiento empresarial.

Luego del procesamiento, análisis e interpretación de los datos obtenidos, podemos determinar que las empresas necesitan implementar un modelo de gestión empresarial que se ajuste a su giro de negocios y que se organicen considerando tanto factores internos como externos con el fin de poder mejorar la calidad de sus productos y/o servicios y alcanzar el cumplimiento de las metas propuestas.

El modelo de Malcom Baldrige como lo indican Silva et al. (2020), es un modelo que se enfoca en el bienestar interno de la empresa apoyando a la capacitación y formación de un talento humano eficiente y capaz, además 
da gran importancia a la satisfacción de los clientes generando así mayor productividad, por otra parte Recalde et al. (2017) considera que el modelo Deming, se centra en la correcta administración y organización de los procesos de la empresa, es decir lleva una planificación de actividades luego se ejecutan para luego realizar su respectiva evaluación y por último se toman decisiones o medidas para mejorar continuamente el desarrollo de actividades en la empresa.

El modelo administrativo Servqual según Pincay y Parra (2020), se enfoca en la medición o evaluación de la calidad de servicio mediante la valoración del cliente ante el producto o servicio ofertado por la empresa, de esta manera se conoce el nivel de aceptación y confianza del consumidor con la finalidad de fidelizar al mismo, es decir es un modelo que se enfoca en las necesidades y expectativas del cliente.

Siguiendo el objetivo del presente estudio, el modelo Deming es el más adecuado, ya que es un modelo que se centra específicamente en la forma de administrar de las empresas partiendo desde la planificación de cada actividad, ejecución de estas, evaluación de cada proceso realizado y así mismo un factor importante es las medidas o decisiones que se toman en base a los resultados obtenidos, generando estrategias que permitan a las empresas crecer y cumplir objetivos. De esta manera las empresas que no poseen planificación en sus actividades además no cuentan con una filosofía y estructura empresarial adaptada a su realidad, tengan una visión clara de la importancia y cambio que genera su aplicación, ya que contribuye a la organización empresarial ayudando a la toma correcta de decisiones y generación de estrategias de mejora continua para lograr el crecimiento esperado en la empresa.

\section{Conclusiones}

Finalizando con la investigación se logró determinar en base a los resultados que gran parte de las empresas objeto de estudio, carecen de una organización adecuada, debido a factores como, la ausencia de estructura y filosofía empresarial, falta de planificación de actividades y adecuado control y gestión de procesos, para lo que es necesaria la aplicación de una herramienta que permita la organización de procesos y actividades que apoyen al logro de objetivos y crecimiento empresarial.

Mediante el análisis del presente estudio se determinó que el modelo Deming, es el modelo que se más se adapta a las necesidades de las MIPYMES de la ciudad de Machala, dado el modelo en mención se centra en la forma de administrar las empresas partiendo de una planificación de actividades para su posterior ejecución y evaluación que en contraste con el Modelo de Malcom y Baldrige cuyo enfoque es la mejora del talento humano, este busca crear una sinergia para el correcto actuar de la empresa.

Finalmente, a través de este estudio se logró determinar que la aplicación de los modelos administrativos y la organización empresarial pueden ser considerados como estrategia para el crecimiento a mediano plazo en las MIPYMES de la ciudad de Machala, porque permiten una estructuración que se adapta a la necesidad de la empresa además de dar apoyo a la gestión, supervisión y control de actividades, recursos, inventario y talento humano, alcanzando así el logro de los objetivos propuestos.

\section{Referencias bibliográficas}

Vega, L. (2016). Las Nuevas Formas de Organización y la Empresa Socialmente Responsable, Piezas fundamentales para el desarrollo de la innovación. TEACS, 31 - 45. Obtenido de https://www.redalyc. org/pdf/993/99344833008.pdf

Armijos, L. (2019). CAUSAS Y CONSECUENCIAS DEL FRACASO EMPRESARIAL: UN ANALISIS DESDE LA COMPETITIVIDAD EMOCIONAL. Tambara, 230-242. Obtenidodehttps://www.researchgate.net/ publication/337845427_CAUSAS_Y CONSECUENCIAS DEL FRACA $\bar{S} O$ EMPRESARIAL_UN_ $\bar{A} N A L I S I S-$ DESDE LA COMPETITIVIDAD EMOCIONAL_CAUSES_AND CONSEQUENCESSOF_BUSINESS FAILURE_AN_ANALYSIS_FROM_ 


\section{EMOTIONAL_COMPETITIVENESS}

Arteaga, H., Intriago, D., \& Mendoza, K. (6 de julio de 2016). La ciencia de la administración de empresas. Dominio de las ciencias, 421-431. Obtenido de https://dialnet.unirioja.es/descarga/ articulo/5802887.pdf

Baque, M., Chele, J., Cedeño, B., \& Gaona, V. (2020). Fracaso de las pymes: Factores desencadenantes, Ecuador 2020. FIPCAEC, 5-25. doi:DOI: https://doi. org/10.23857/fipcaec.v5i4.293

Bernal, E., Erazo , J., \& Narváez, C. (2019). Estructuras organizativas favorables a la Eficiencia Empresarial. Revista Arbitrada Interdisciplinaria KOINONIA, 3-31. doi:DOI: $10.35381 /$ r.k.v4i1.370

Buñay, J., Montalvan, J., \& Vizueta Sonia. (febrero de 2018). Administración tradicional vs el Toyotismo: Una formula efectiva para las PyMES. Mundo de la Investigacion y el Conocimiento, 464-487. doi:10.26820/ recimundo/2.1.2018.464-487

CEPAL. (2018). MIPYMES en America Latina Un frágil desempeño y nuevos desafíos para las políticas de fomento. cepal. Obtenido de https://repositorio.cepal. $\mathrm{org} / \mathrm{bitstream} / \mathrm{handle} / 11362 / 44148 / 1 /$ S1800707_es.pdf

Cervantes, X., \& Oviedo, B.(2018). LAS MICRO, PEQUEÑAS, MEDIANAS EMPRESAS DEL SECTOR MANUFACTURERO: UN ESTUDIO DEL CONTEXTO DE LA GESTIÓN POR PROCESOS EN EL CANTÓN EL EMPALME, ECUADOR. | Revista Cientifica de la Universidad de Cienfuegos, 366-371. Obtenido de http:// scielo.sld.cu/pdf/rus/v10n5/2218-3620rus-10-05-366.pdf

Flores, L. (2017). GESTIÓN DEL TALENTO HUMANO Y SU INCIDENCIA EN LA CALIDAD DE SERVICIO Y RENTABILIDAD DE LAS EMPRESAS DE TRANSPORTE TERRESTRE DE PASAJEROS INTERREGIONAL DEL SUR DEL PERÚ, PERIODO 20152016. REVISTA DE INVESTIGACIONES DE LA ESCUELA DE POSGRADO,
348-36. doi:http://dx.doi.org/10.26788/ riepg.2017.4.59

Hernández , H., Martinez, D., \& Barrios, I. (2018). Gestión de la calidad: elemento clave para el desarrollo de las organizaciones. Criterio Libre, 179-195. Obtenido de https://core.ac.uk/download/ pdf/229911313.pdf

Hernández , R., Fernández, C., \& Baptista, M. (2014). Metodología de la Investigación. México D.F.: McGRAW-HILL.

INEC. (2015). Censo sobre MIPYMES. Instituto Nacional de Estadísticas y Censos. INEC, http://www.ecuadorencifras.gob.ec.

INEC. (2019). Directorio de empresas $\mathrm{y}$ establecimientos 2018. INEC, 1--46. Obtenido de https://www. ecuadorencifras.gob.ec/documentos/ web-inec/Estadisticas Economicas/ DirectorioEmpresas/Directorio Empresas_2018/Principales_Resultados_ DIEE_2018.pdf

Larios, E. (2016). La Gestión de la Competitividad en la MIPYME Mexicana: Diagnóstico Empírico desde la Gestión del Conocimiento. redalyc, 177-209. Obtenido de https://www.redalyc.org/ pdf/2737/273746863008.pdf

Luciani, L., Zambrano, A., \& Gonzalez, A. (2019). MIPYMES ecuatorianas: Una visión de su emprendimiento, productividad y competitividad en aras de mejora continua. Cooperativismo y desarrollo, 313-332. Obtenido de http:// coodes.upr.edu.cu/index.php/coodes/ article/view/217/430

Navarro , A., Cota , R., \& González, C. (2018). CONCEPTOS PARA ENTENDER LA INNOVACIÓN ORGANIZACIONAL. Revista de Comunicación de la SEECI, 87-101. doi:http://doi.org/10.15198/ seeci.2018.45.87-101

Ocampo, W., Huilcapi, N., \& Cifuentes, A. (2019). La estructura organizacional en función del comportamineto del capital humano en las organizaciones. Revista Cientifica Mundo de la Investigación y el Conocimiento., 114-137. doi:10.26820/ 
recimundo/3.(4).diciembre.2019.114-137

Pincay, Y., \& Parra, C. (2020). Gestión de la calidad en el servicio al cliente de las PYMES comercializadoras. Una mirada en Ecuador. Dominio de las Ciencias, 11181142. doi:http://dx.doi.org/10.23857/ dc.v6i3.1341

Quispe, A., Padilla, M., Telot, J., \& Nogueira, D. (2017). Tecnologías de información $\mathrm{y}$ comunicación en la gestión empresarial de pymes. redalyc, 81-92. Obtenido de https://www.redalyc.org/ pdf/3604/360450397008.pdf

Recalde, M., Paez, J., Yepez, J., \& Andrade , M. (2017). LOS MODELOS DE GESTIÓN ADMINISTRATIVA DE LAS PEQUEÑAS Y MEDIANAS EMPRESAS EN LA CIUDAD DE QUITO. eumednet, $1-27$.

Saltos , M., Muñoz, E., \& Rodriguez, L. (2016). Modelo de Organización por Procesos - Caso de Ejemplificación en una Institución Pública. Publicando, 236-256. Obtenido de https://revistapublicando.org/ revista/index.php/crv/article/viewFile/86/ pdf_130

Sanchez, M., Cervantes, V., \& Peralta, P. (2016). Gestión de la innovación en pequeñas $\mathrm{y}$ medianas empresas de Barranquilla - Colombia. redalyc, 78-91. Obtenido de https://www.redalyc.org/ pdf/280/28049145007.pdf

Silva, J., Feijó, N., \& Feijoo, T. (2020). MODELOS DE GESTIÓN DE CALIDAD PARA EL DESARROLLO DE EMPRENDIMIENTOS EN LAS INSTITUCIONES DE EDUCACIÓN SUPERIOR DE MANABÍ. Revista Cientifica Multidisciplinaria Arbitrada YACHASUN(ISSN: 2697-3456), 152-163. doi:https://doi.org/10.46296/yc.v4i7.0039

Taillacq, D., Álvarez, H., \& Blanco, C. (2016). PROCEDIMIENTO PARA LA EVALUACIÓN DE LA CALIDAD PERCIBIDA DE LOS SERVICIOS DE RESTAURACIÓN NO ESTATALES CONTRATADOS POR LA AGENCIA HAVANATUR S.A. Revista Cientifica de la Universidad de Cienfuegos, 20-32. Obtenido de http://scielo.sld.cu/pdf/rus/ v8n2/rus02216.pdf

Texis, M., Ramirez , M., \& Aguilar, J. (2016). Microempresas de base social y sus posibilidades de supervivencia. scielo, 551-567. doi:https://doi.org/10.1016/j. cya.2015.04.001

Valarezo, C., \& Coronel, B. (2018). ADMINISTRACIÓN HOTELERA, FLEXIBILIDADPARALAEFICIENCIA. Economia Latinoamericana, 1-9. Obtenido de https://www.eumed.net/rev/ oel/2018/08/administracion-hotelera.html

Vega, L., \& Salaiza , F. (2016). Nuevas formas de organización para la innovación administrativa y técnica. Telos, 121-137. Obtenido de https://www.redalyc.org/ pdf/993/99344833008.pdf

Veliz, A. (2018). La Comunicación como medio de retroalimentación en la Administración de Empresas. polo del conocimiento, 93103. doi:10.23857/casedelpo.2018.3.2.feb rero.93-103

Viloria, N., \& Luciani, L. (2015). El pensamiento administrativo: Un estudio de sus ejes problemáticos. Sapienza Organizaciona, 119-143. Obtenido de https://www. redalyc.org/pdf/5530/553056600008.pdf 